\title{
Mobile Phone Programming for Multimedia
}

\author{
Jürgen Scheible \\ Media Lab \\ University of Art and Design, Helsinki \\ Hämeentie 135C, FI-00560 Helsinki, \\ Finland \\ +358405509268 \\ jscheib@uiah.fi
}

\begin{abstract}
This compact hands-on tutorial introduces a novel way of creative mobile phone programming for multi-media that is easy to learn and fun. It was created in 2006 by the author of this paper and was published as a free online tutorial at http://www.mobilenin.com/pys60/menu.htm. Pedagogically finetuned, it teaches the programming of a large set of mobile phone features including camera, sound, video, messaging, telephony, location, Bluetooth, graphics, Wi-fi, GPS and networking. The problem in the past has been that developing applications on the mobile platform was time consuming and required a steep learning curve. Also, mobile platforms have often been closed or were too restricted. Mobile Python, also known as 'Python for S60' (PyS60) offers a crucial turning point here. It provides a Python execution environment for the mobile phone. It is developed by Nokia Research as an Open Source Project. This tutorial uses PyS60 in combination with a set of code examples, turning it into a ready working toolkit. It allows developing mobile applications even by novice programmers, artists and people from the creative communities. In a matter of days, people can build powerful applications based on their own ideas and contribute to the mobile space. This tutorial has been taught in more than 40 occasions in academia and industry and in more than 17 countries during the last 2 years. Among these are Yahoo Research Berkeley, Nokia, NTU Taiwan, MIT and Stanford University.
\end{abstract}

\section{Categories and Subject Descriptors}

K.3.2 [Computers and Education]: Computer and Information Science Education - Computer science education, Curriculum, Self-assessment. D.1.5 [Programming Techniques]: Objectoriented Programming. H.5.2 [Information Interfaces and Presentation]: User Interfaces - Prototyping

\section{General Terms}

Design, Experimentation, Human Factors.

\section{Keywords}

Mobile Python, Tutorial, Python for S60, PyS60.

Copyright is held by the author/owner(s).

$M M^{\prime} 07$, September 23-28, 2007, Augsburg, Bavaria, Germany.

ACM 978-1-59593-701-8/07/0009.

\section{INTRODUCTION}

The mobile space and the internet are rapidly converging and are turning into a rich source of opportunities. There exists a need for research and innovation in this field. Whether you want to create an application that takes a photo, records sound or video for instant sharing with your friends or for uploading to a blog, or whether you want to turn the phone into a device for interacting with objects like public screens, shop windows or smart fashion (wearables) or even want to create games with 2D and 3D graphics, or design location based applications using the built-in GPS reader of the phone. Many of such applications can be created in a fast an efficient manner using Mobile Python. See video demos at http://www.mobilenin.com. Some projects have been reported also to the Research community such as e.g. 'Manhattan Story Mashup' [1] or 'MobiLenin' [2].

\section{TUTORIAL OUTLINE}

The tutorial is tailored for MM '07 but is based on the online tutorial at http://www.mobilenin.com/pys60/menu.htm.

- Introduction to PyS60

- A trigger for innovation and a toolkit for rapid prototyping

- Feature overview and demo examples

- Hands-on session

- GUI programming, SMS sending/receiving

- Sound recording/playing, Midi, Text to speech, OSC

- Camera, 2D\& 3D Graphics, Keyboard keys, Video playing

- Bluetooth (phone to phone, phone to computer, phone to microcontroller e.g. controlling Max/MSP or Arduino board)

- Networking: File up/download, Wi-fi, client-server apps

- Location, Contacts and Calendar

- GPS position data (built-in GPS reader)

Prerequisites. All you need is some basic programming or scripting knowledge (like JavaScript, Lingo, Flash AS, ...). No Python or mobile platform knowledge is needed.

The teaching approach. The approach taken in this tutorial is hands-on, meaning participants first hear the explanation to some code, and then use their computer to write the Python code [3] in form of a script and then move it to the phone for instant testing. This provides strong feedback and high motivation for learning. 
Usually all features that PyS60 offers can be learned within 2-3 days. This half day tutorial will be a condensed session.

\section{WHAT IS PYTHON FOR S60}

Python for S60 (PyS60) brings the Python programming language (http://www.python.org) to the S60 Platform (only), which is Nokia's smartphone platform based on Symbian OS. By installing a freely available Python interpreter (named Python for S60) to the phone, Python scripts can be executed, providing access to many API's of the smartphone (http://sourceforge.net/projects/pys60).

PyS60 is easy to learn and makes development of mobile applications fast with completely free and open tools and by reusing open source code modules. It makes rapid prototyping efficient by wrapping complex low-level technical details behind simple interfaces. And above all - it makes programming on the mobile platform fun. PyS60 has been adopted by many Universities and research projects already, as well as by companies. The use of PyS60 and is on the rise.

Simple code example. The following three lines of code build a fully working script that displays a text input field (figure 1a) waiting for the users input and then shows a popup note (figure 1b) as the result:

import appuifw

data = appuifw.query(u"Type your name:", "text")

appuifw.note(u"Greetings from: " +data)

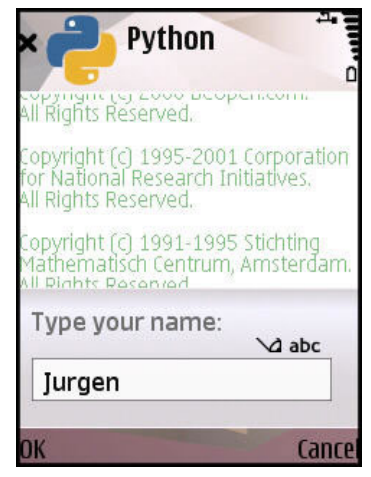

(a)

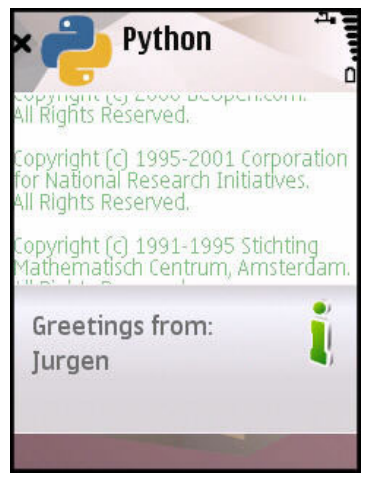

(b)
Figure 1. Screenshots of a simple PyS60 application. (a) Text input field; (b) Pop-up note;

User driven innovation. When running workshops in many places and countries using this tutorial, I have seen that many people innovate instantly, they create unique and novel applications based on their own ideas fulfilling their own needs and they want to share their innovation. This practical experiences matches well with Eric von Hippel's theory of user driven innovation [4]. He talks in his book about Democratizing Innovation and the phenomena that users can generate innovation if a tool kit is given into their hands. He calls this distributed innovation by so called Lead users. This raises the question whether Mobile Python as a toolkit could lead into an era of 'democratizing innovation on the mobile platform'?
The vision. Wouldn't it be great to see a big garden full of beautiful flowers, each representing a novel mobile application created by a lead user, fitting her own needs? My Message to all the creative and innovative people out there: Use your talent, skills, ideas and energy to inspire the world! May this tutorial help you to do so!

Mobile Python book. For those interested in boosting their knowledge on mobile phone programming for multi-media, based on the approach of this tutorial, there is also a book available with the title 'Mobile Python - Rapid prototyping on the Mobile platform' [5].

\section{SPEAKER'S BIOGRAPHY}

Jürgen Scheible is a music and media artist. He is a doctoral student at the Media Lab, University of Art and Design, Helsinki where he runs the Mobile Hub, a prototype development environment for mobile client/server applications with a strong focus on artistic approaches and creative design. He spent several months in 2006 as a visiting scientist at MIT, CSAIL, Boston and worked previously for Nokia for 8 years. In 2006 and 2007 Jürgen was recognized as a Forum Nokia Champion for his driving vision to be a bridge builder between art, engineering and research. He is internationally active in teaching innovation workshops on rapid mobile application prototyping in academic but also professional settings e.g. at Stanford University, MIT, NTU Taiwan, Yahoo Research Berkeley, Nokia. He was one of the ACM Computers in Entertainment Scholarship Award winners in 2006 and Best Arts Paper Award winner at ACM Multimedia 2005 conference. His research focuses on designing multi-modal user interfaces for creating and sharing interactive artistic experiences.

\section{ACKNOWLEDGMENTS}

I would like to thank Ville Tuulos for his invaluable contributions for improving the tutorial, and Jukka Laurila for his great work he is doing for PyS60.

\section{REFERENCES}

[1] Tuulos V., Scheible J. and Nyholm H., Combining Web, Mobile Phones and Public Displays in Large-Scale: Manhattan Story Mashup. In Proceedings of the Fifth International Conference on Pervasive Computing, Toronto, Canada (May 2007).

[2] Scheible, J., Ojala, T.: MobiLenin - Combining a multi-track music video, personal mobile phones and a public display into multi-user interactive entertainment. In: MULTIMEDIA '05: Proceedings of the 13th annual ACM international conference on Multimedia, Singapore, ACM Press (Nov. 2005), 199-208.

[3] Lutz M., Ascher D. Learning Python. O'Reilly, 2004.

[4] Von Hippel E. Democratizing Innovation. MIT Press, 2005.

[5] Scheible J., Tuulos V. Mobile Python - Rapid Prototyping on the Mobile Platform. Wiley, 2007. 